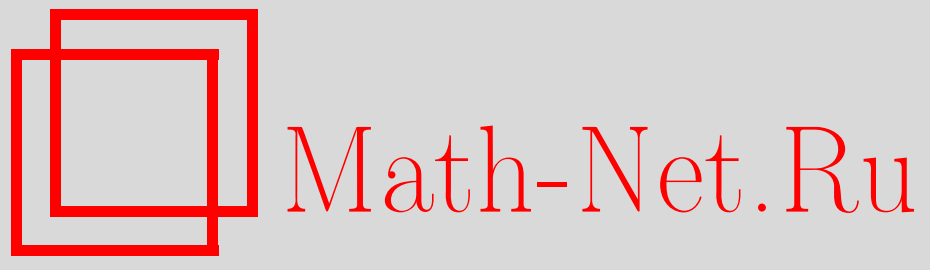

А. Н. Тимашёв, Об асимптотике больших уклонений в схемах размещения частиц по различным ячейкам при ограниченных объемах ячеек, Теория вероятн. и ее примен., 2000, том 45, выпуск 3, 521-535

DOI: https://doi.org/10.4213/tvp483

Использование Общероссийского математического портала Math-Net.Ru подразумевает, что вы прочитали и согласны с пользовательским соглашением http: //www . mathnet.ru/rus/agreement

Параметры загрузки:

IP: 3.81 .55 .215

26 апреля 2023 г., 15:35:45

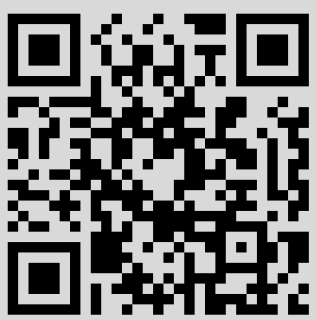




\title{
ОБ АСИМПТОТИКЕ БОЛЬШИХ УКЛОНЕНИЙ В СХЕМАХ РАЗМЕЩЕНИЯ ЧАСТИЦ ПО РАЗЛИЧНЫМ ЯЧЕЙКАМ ПРИ ОГРАНИЧЕННЫХ ОБЪЕМАХ ЯЧЕЕК
}

\begin{abstract}
Рассматриваются равновероятные схемы размещения $\boldsymbol{n}$ одинаковых и различных частиц по $N$ различным ячейкам при условии, что количество частиц, содержащихся в каждой ячейке, не превосходит некоторой постоянной $p \in \mathbf{N}$. Получены локальная и интегральная теоремы о больших уклонениях, оценивающие хвосты распределения случайной величины, равной числу пустых ячеек. Найдены асимптотики математического ожидания и дисперсии и доказана локальная нормальная теорема для распределения вероятностей этой случайной величины в центральной зоне изменения параметров $n, N$, когда $n, N \rightarrow \infty$ так, что $0<\alpha_{1} \leqslant \alpha=n / N \leqslant \alpha_{2}<p$ $\left(\alpha_{1}, \alpha_{2}\right.$ - постоянные).
\end{abstract}

Ключевые слова и фразы: равновероятная схема размещения, локальная предельная теорема, большие уклонения, метод перевала.

1. Введение. Пусть $n-$ количество частиц, $N$ - количество ячеек в равновероятной схеме размещения различных частиц по различным ячейкам при условии, что объем (т.е. количество содержащихся частиц) каждой ячейки не превосходит $p$. Всюду далее $p-$ фиксированное натуральное число и $n \leqslant N p$. Положим

$$
M_{n}(N, p)=\sum_{\substack{l_{1}+\cdots+l_{N}=n \\ 0 \leqslant l_{1}, \cdots, l_{N} \leqslant p}} \frac{n !}{l_{1} ! \cdots l_{N} !} .
$$

Будем предполагать, что все $M_{n}(N, p)$ размещений указанного типа имеют одинаковые вероятности, равные $\left(M_{n}(N, p)\right)^{-1}$. Если $\nu_{i}$ - объем $i$-й ячейки, $i=1,2, \ldots, N$, и $k_{1}, \ldots, k_{N}$ - целые неотрицательные числа, для которых $k_{1}+\cdots+k_{N}=n$ и $k_{i} \leqslant p ; i=1,2, \ldots, N$, то

$$
\mathbf{P}\left\{\nu_{1}=k_{1}, \ldots, \nu_{N}=k_{N}\right\}=\frac{1}{M_{n}(N, p)} \frac{n !}{k_{1} ! \cdots k_{N} !} .
$$

Пусть $\mu_{0}(p)$ - случайная величина, равная количеству пустых ячеек в рассматриваемой схеме случайных размещений, тогда $N-n / p \geqslant \mu_{0}(p) \geqslant$

\footnotetext{
*Академия ФСБ, Мичуринский пр., 70, 125040 Москва, Россия.
} 
$N-n$ с вероятностью 1 . В частности, при $p=1$ из (1) следует, что $M_{n}(N, 1)=N(N-1) \cdots(N-n+1)$ и $\mu_{0}(1)=N-n$ с вероятностью $1 ;$ в этом случае получаем хорошо известную схему выбора без возврашения $[1$, с. 40$]$. Если $n \leqslant p$, то $M_{n}(N, p)=N^{n}$, и мы получаем классическую схему равновероятных размешений, исследовавшуюся многими авторами (см. обобщающую монографию [2]). Задача оценки распределения случайной величины $\mu_{0}(p)$ в этой схеме носит название классической задачи о дробинках $[2$, с. 9$]$.

Известно [3, с. 123], что производящая функция чисел $M_{n}(N, p)$ имеет следующий вид $\left(M_{0}(N, p)=1\right)$ :

$$
\sum_{n=0}^{N p} M_{n}(N, p) \frac{z^{n}}{n !}=\left(\sum_{k=0}^{p} \frac{z^{k}}{k !}\right)^{N} .
$$

Из (3) получаем

$$
M_{n}(N, p)=\frac{n !}{2 \pi i} \oint \frac{\left(1+z+\cdots+z^{p} / p !\right)^{N}}{z^{n+1}} d z .
$$

Интегрирование в (4) осуществляется по окружности произвольного радиуса с центром в нуле, пробегаемой в положительном направлении.

В работе, исходя из (4) и с использованием метода перевала (в одномерном и двумерном вариантах) получены локальная и интегральная теоремы о больших уклонениях, опениваюие хвосты распределения вероятностей случайной величины $\mu_{0}(p)$ в центральной зоне изменения параметров $n, N[2$, с. 13$]$, когда $n, N \rightarrow \infty$ так, что

$$
0<\alpha_{1} \leqslant \alpha=\frac{n}{N} \leqslant \alpha_{2}<p
$$

( $\alpha_{1}, \alpha_{2}$ - постоянные). Заметим, что в классической схеме равновероятных размещений локальная и интегральная многомерные теоремы о больших уклонениях получены автором в [4], [5].

Применяя полученные результаты, в работе найдена асимптотика математического ожидания и дисперсии случайной величины $\mu_{0}(p)$ при условии (5), а также доказана локальная нормальная предельная теорема для распределения $\mu_{0}(p)$. При этом используется метод сведения к суммам независимых одинаково распределенных целочисленных случайных величин, впервые реализованный В. Ф. Колчиным [2, с. 58$]$.

Далее рассматривается равновероятная схема размещения $n$ одинаковых частиц по $N$ различным ячейкам при указанном выше ограничении на объемы ячеек. Пусть $f_{n}(N, p)$ - число всевозможных размешений такого типа, т.е. число решений уравнения $x_{1}+\cdots+x_{N}=n$ в целых неотрицательных числах $x_{1}, \ldots, x_{N}$ таких, что $x_{i} \leqslant p, i=1,2, \ldots, N$.

Предполагается, что все $f_{n}(N, p)$ размещений имеют одинаковые вероятности, равные $\left(f_{n}(N, p)\right)^{-1}$. Пусть $\xi_{0}(p)-$ случайная величина, 
равная количеству пустьх ячеек в этой схеме случайных размещений, тогда $N-n / p \geqslant \xi_{0}(p) \geqslant N-n$ с вероятностью 1 . В частности, при $p=1$ $f_{n}(N, 1)=C_{N}^{n} ; \xi_{0}(1)=N-n$ с вероятностью 1 , и мы получаем известную статистику Ферми-Дирака $[1$, с. 52$]$. Если $n \leqslant p$, то $f_{n}(N, p)=C_{n+N-1}^{N-1}$, и мы получаем статистику Бозе-Эйнштейна $[1$, с. 52]. Предельные теоремы для распределения числа ячеек с заданным заполнением в этой схеме получены А.Н. Труновым в [6]; теоремы о больших уклонениях доказаны автором в [7].

Известно [3, с. 126], что производящая функция чисел $f_{n}(N, p)$ имеет вид

$$
\sum_{n=0}^{N p} f_{n}(N, p) z^{n}=\left(\sum_{k=0}^{p} z^{k}\right)^{N}
$$

Из (6) следует, что

$$
f_{n}(N, p)=\frac{1}{2 \pi i} \oint \frac{\left(1+z+\cdots+z^{p}\right)^{N}}{z^{n+1}} d z .
$$

Интегрирование в (7) осуществляется по тому же пути, что и в (4).

Исходя из (7) и используя метод перевала при условии (5), в работе получены локальная и интегральная теоремы о больших уклонениях, асимптотика математического ожидания и дисперсии, а также локальная нормальная предельная теорема для распределения случайной величины $\xi_{0}(p)$.

В теоремах 1 и 7 получены асимптотические оценки чисел $M_{n}(N, p)$ и $f_{n}(N, p)$ всевозможных размещений указанных типов, определяемых согласно (4) и (7), при условии, что $n, N \rightarrow \infty$ так, что выполнено (5). При этом остаточный член в обоих случаях имеет порядок $O(1 / N)$. В теоремах 2 и 8 при условии (5) доказываются асимптотические формулы для вероятностей $\mathbf{P}\left\{\mu_{0}(p)=m\right\}$ и $\mathbf{P}\left\{\xi_{0}(p)=m\right\}$, если $m \rightarrow \infty$ так, что выполнено (16) (см. ниже), а в теоремах 3,4 и 9,10 - асимптотические формулы для вероятностей $\mathbf{P}\left\{\mu_{0}(p) \geqslant m\right\}, \mathbf{P}\left\{\mu_{0}(p) \leqslant m\right\}$ и $\mathbf{P}\left\{\xi_{0}(p) \geqslant m\right\}$, $\mathbf{P}\left\{\xi_{0}(p) \leqslant m\right\}$. При этом во всех случаях остаточный член также имеет порядок $O(1 / N)$, а параметр $m$ может попадать и в области больших уклонений. В теоремах 5 и 11 находятся асимптотические оценки для математических ожиданий $\mathbf{E} \mu_{0}(p), \mathbf{E} \xi_{0}(p)$ и дисперсий $\mathbf{D} \mu_{0}(p), \mathbf{D} \xi_{0}(p)$ при условии (5); для вычисления дисперсий в обоих случаях приходится использовать точную формулу для остаточного члена порядка $O(1 / N)$ в асимптотических разложениях величин $M_{n}(N, p)$ и $f_{n}(N, p)$. Наконец, в теоремах 6 и 12 формулируются и доказываются утверждения о сходимости распределений случайных величин $\mu_{0}(p)$ и $\xi_{0}(p)$ к нормальному закону (в локальном варианте), из которых обычным образом следуют интегральные теоремы. Формулировки и доказательства всех изложенных результатов для распределения случайной величины $\xi_{0}(p)$ анало- 
гичны соответствуюшим результатам для $\mu_{0}(p)$, поэтому теоремы 7-12 только формулируются. Заметим, что при выводе локальных и интегральных теорем о больших уклонениях (как и в классических схемах размещения - см. [4], [5], [7]) не удается обойтись чисто вероятностными средствами исследования, что обуславливает необходимость использования многомерного метода перевала (в техническом отношении довольно сложного). Однако, в отличие от указанных классических схем, в рассматриваемых в работе случаях пока что не удалось получить ни одной пуассоновской предельной теоремы для случайных величин $\mu_{0}(p)$ и $\xi_{0}(p)$.

\section{2. Случай, когда частицы различны.}

Теорема 1. Пусть $n, N \rightarrow \infty$ так, ито выполнено условие (5). Тогда равномерно относительно $\alpha \in\left[\alpha_{1}, \alpha_{2}\right]$

$$
M_{n}(N, p)=\frac{n ! \beta_{0}^{-N} z_{0}^{-n}}{\sqrt{2 \pi N\left(\alpha-\left(z_{0}-\alpha\right)(p-\alpha)\right)}}\left(1+O\left(\frac{1}{N}\right)\right),
$$

где $z=z_{0}-$ единственный веществениьй положительный корень уравнения

$$
\sum_{k=0}^{p}(k-\alpha) \frac{z^{k}}{k !}=0
$$

$u$

$$
\beta_{0}=\beta_{0}(\alpha)=\left(\sum_{k=0}^{p} \frac{z_{0}^{k}}{k !}\right)^{-1}=\frac{p !\left(z_{0}-\alpha\right)}{z_{0}^{p+1}} .
$$

Д ок аз а т е ль с т в о. Согласно (4), имеем:

$$
M_{n}(N, p)=\frac{n !}{2 \pi i} \oint \frac{1}{z} \exp (N \omega(z)) d z,
$$

где $\omega(z)=\ln \left(1+z+\cdots+z^{p} / p !\right)-\alpha \ln z$ и для логарифма выбирается та непрерывная ветвь, которая принимает вещественные значения на вещественной положительной полуоси.

Следовательно, $\omega^{\prime}(z)=0$, если $z=z_{0}$ - единственный вещественный положительный корень уравнения (9), и $\omega^{\prime \prime}\left(z_{0}\right)=\left[\alpha-\left(z_{0}-\alpha\right)(p-\right.$ $\alpha)] / z_{0}^{2}>0[8$, c. 192]. При условиях теоремы 1 значение интеграла в (4) асимптотически, с точностью до множителя $1+O(1 / N)$, равно вкладу точки перевала первого порядка $z=z_{0}$ функции $\omega$, и притом равномерно относительно $\alpha \in\left[\alpha_{1}, \alpha_{2}\right]$; вычисляя этот вклад стандартными методами $\left[8\right.$, c. 194], приходим к соотношению (8), в котором величина $\beta_{0}$ определяется из (10). Теорема 1 доказана.

Заметим, что если положить

$$
S_{r}(z)=\sum_{k=0}^{r} \frac{z^{k}}{k !} \quad(z>0, r=0,1, \ldots),
$$


то окажется, что уравнение (9) можно записать в виде $z S_{p-1}(z) / S_{p}(z)=$ $z S_{p}^{\prime}(z) / S_{p}(z)=\alpha$; при этом функция $q(z)=z S_{p}^{\prime}(z) / S_{p}(z)$ строго возрастает от 0 до $p$ при возрастании $z$ от 0 до $+\infty[8$, c. 191]. Поэтому, полагая для любого $\alpha \in(0, p) z_{0}=z_{0}(\alpha)$, получаем, что функция $z_{0}(\alpha)$ строго возрастает от 0 до $+\infty$ при возрастании $\alpha$ от 0 до $p$. Отсюда, в частности, следует, что $\alpha<z_{0}(\alpha)<\alpha(1+1 /(p-\alpha))$ для любого $\alpha \in(0, p)$.

Рассмотрим случайную величину $\mu_{0}(p)$, определенную выше во введении. Если случайный вектор $\left(\nu_{1}, \nu_{2}, \ldots, \nu_{N}\right)$ имеет распределение вида $(2)$, то $\mu_{0}(p)=\operatorname{card}\left\{i \in \mathbf{N} \mid 1 \leqslant i \leqslant N, \nu_{i}=0\right\}$.

Факториальные моменты случайной величины $\mu_{0}(p)$ определяются из соотношений

$$
\mathbf{E}\left(\mu_{0}(p)\right)_{j}=N(N-1) \cdots(N-j+1) \frac{M_{n}(N-j, p)}{M_{n}(N, p)}
$$

$\left(j=0,1, \ldots, N, M_{n}(0, p)=0\right)$. Из (4) и (12) следует, что производящая функция распределения вероятностей для $\mu_{0}(p)$ равна

$$
\begin{aligned}
P_{\mu_{0}(p)}(u) & =\sum_{m=0}^{N} \mathbf{P}\left\{\mu_{0}(p)=m\right\} u^{m} \\
& =\frac{n !}{M_{n}(N, p)} \frac{1}{2 \pi i} \oint \frac{\left(u+\sum_{k=1}^{p} z^{k} / k !\right)^{N}}{z^{n+1}} d z .
\end{aligned}
$$

По интегральной формуле Коши имеем, согласно (13),

$$
\mathbf{P}\left\{\mu_{0}(p)=m\right\}=\frac{n !}{M_{n}(N, p)} \frac{1}{(2 \pi i)^{2}} \oint \oint \frac{(f(z, u))^{N}}{z^{n+1} u^{m+1}} d z d u
$$

$(m=0,1, \ldots, N)$, где

$$
f(z, u)=u+\sum_{k=1}^{p} \frac{z^{k}}{k !}
$$

Всюду далее считаем, что $m$ - натуральное число.

Теорема 2. Пусть $n, N \rightarrow \infty$ так, ито выполнено условие (5); $p \geqslant 2 u m=\beta N \rightarrow \infty, \max$, umo

$$
1<\gamma_{1} \leqslant \frac{\alpha}{1-\beta} \leqslant \gamma_{2}<p \quad\left(\gamma_{1}, \gamma_{2}-\text { постоянные }\right) .
$$

Тогда равномерно относительно $\alpha \in\left[\alpha_{1}, \alpha_{2}\right]$ и значений $\beta$, удовлетворяющих условию (16),

$$
\begin{aligned}
\mathbf{P}\left\{\mu_{0}(p)=m\right\}= & \sqrt{\frac{\alpha-\left(z_{0}-\alpha\right)(p-\alpha)}{2 \pi N \Delta_{z}}}\left(\frac{z_{0}}{z^{*}}\right)^{n} \\
& \times \beta_{0}^{N} \beta^{-m}\left(\frac{S_{p}\left(z^{*}\right)-1}{1-\beta}\right)^{N-m}\left(1+O\left(\frac{1}{N}\right)\right),
\end{aligned}
$$


где $z=z_{0}$ - единственный вещественный положительный корень уравнения (9); $z=z^{*}-$ единственный вещественный положительный корень уравнения

$$
\begin{gathered}
\sum_{k=1}^{p}\left(k-\frac{\alpha}{1-\beta}\right) \frac{z^{k}}{k !}=0 \\
\Delta_{z}=\beta(1-\beta)^{2}\left(\frac{z^{*}\left(S_{p-1}\left(z^{*}\right)+z^{*} S_{p-2}\left(z^{*}\right)\right)}{S_{p}\left(z^{*}\right)-1}-\frac{\alpha^{2}}{(1-\beta)^{2}}\right)>0
\end{gathered}
$$

значения $\beta_{0}, S_{p-2}\left(z^{*}\right), S_{p-1}\left(z^{*}\right), S_{p}\left(z^{*}\right)$ определяются согласно (10), (11).

Д ок а з а т е л ь с т в о. Для обоснования формулы (17) воспользуемся методом перевала, исходя из соотношений (14) и (15) и учитывая условия (5) и (16). Пусть $\left(z^{*}, u^{*}\right)$ - точка перевала функции $f$, для которой $z^{*}>0$ и $u^{*}>0$, тогда $\max _{|z|=z^{*},|u|=u^{*}}|f(z, u)|$ достигается только при $z=z^{*}$ и $u=u^{*}$. Чтобы найти значения $z^{*}$ и $u^{*}$, составим систему уравнений

$$
\left\{\begin{array}{l}
N z \frac{\partial f}{\partial z}(z, u)=n f(z, u) \\
N u \frac{\partial f}{\partial u}(z, u)=m f(z, u) .
\end{array}\right.
$$

Подставляя в эту систему $f(z, u)$ из $(15)$ и вычисляя частные производные $(\partial f / \partial z)(z, u)$ и $(\partial f / \partial u)(z, u)$, после элементарных преобразований получаем, что

$$
\sum_{k=1}^{p} \frac{z^{k}}{(k-1) !}=\frac{n}{N-m} \sum_{k=1}^{p} \frac{z^{k}}{k !}=\frac{\alpha}{1-\beta} \sum_{k=1}^{p} \frac{z^{k}}{k !} .
$$

Отсюда следует, что $z=z^{*}$ удовлетворяет уравнению (18), которое при наших условиях имеет единственный вещественный положительный корень $[8$, с. 192$]$. Далее получаем, что

$$
\begin{gathered}
u^{*}=\frac{m}{N-m} \sum_{k=1}^{p} \frac{\left(z^{*}\right)^{k}}{k !}=\frac{\beta}{1-\beta} \sum_{k=1}^{p} \frac{\left(z^{*}\right)^{k}}{k !}, \\
f\left(z^{*}, u^{*}\right)=\frac{1}{1-\beta} \sum_{k=1}^{p} \frac{\left(z^{*}\right)^{k}}{k !} .
\end{gathered}
$$

Из формул (11), (18), (19) и (20) следует, что при условиях теоремы 2

$$
\frac{1}{(2 \pi i)^{2}} \oint \oint \frac{(f(z, u))^{N}}{z^{n+1} u^{m+1}} d z d u=\frac{\left(f\left(z^{*}, u^{*}\right)\right)^{N}}{\left(z^{*}\right)^{n}\left(u^{*}\right)^{m} 2 \pi N \sqrt{\Delta_{z}}}\left(1+O\left(\frac{1}{N}\right)\right)
$$

равномерно относительно $\alpha \in\left[\alpha_{1}, \alpha_{2}\right]$ и значений $\beta$, удовлетворяющих условию (16). Здесь $\Delta_{z}=\operatorname{det}\left(\Delta_{i j}\right)(i, j=1,2)$, значения $\Delta_{i j}$ указаны 
ниже (при этом мы покажем, что $\Delta_{z}>0$ при $p \geqslant 2$ ). Оценки $(8),(14),(21)$ позволяют утверждать, что справедливо равенство (17), где

$$
\begin{aligned}
\Delta_{z} & =\Delta_{11} \Delta_{22}-\Delta_{12} \Delta_{21} \\
\Delta_{11} & =z \frac{\partial}{\partial z}\left(z \frac{\partial(\ln f)}{\partial z}\right)\left(z^{*}, u^{*}\right)=(1-\beta) \frac{\sum_{k=1}^{p}\left[k\left(z^{*}\right)^{k} /(k-1) !\right]}{\sum_{k=1}^{p}\left(z^{*}\right)^{k} / k !}-\alpha^{2}, \\
\Delta_{22} & =u \frac{\partial}{\partial u}\left(u \frac{\partial(\ln f)}{\partial u}\right)\left(z^{*}, u^{*}\right)=\beta(1-\beta), \\
\Delta_{12} & =\Delta_{21}=u \frac{\partial}{\partial u}\left(z \frac{\partial(\ln f)}{\partial z}\right)\left(z^{*}, u^{*}\right)=-\alpha \beta .
\end{aligned}
$$

Следовательно,

$$
\Delta_{z}=\beta(1-\beta)^{2}\left(\frac{\sum_{k=1}^{p} k^{2}\left(z^{*}\right)^{k} / k !}{\sum_{k=1}^{p}\left(z^{*}\right)^{k} / k !}-\frac{\alpha^{2}}{(1-\beta)^{2}}\right)>0
$$

при $p \geqslant 2$; действительно, согласно (18), это неравенство является неравенством Коши-Буняковского и справедливо при $p \geqslant 2$. Кроме того, путем элементарных преобразований нетрудно получить, что величина $\Delta_{z}$ имеет вид, указанный в формулировке теоремы 2. Теорема 2 доказана.

Заметим, что при условиях (5)

$$
\begin{aligned}
1 & <\inf _{\alpha \in\left[\alpha_{1}, \alpha_{2}\right]}\left\{\frac{\alpha}{1-\beta_{0}(\alpha)}\right\}=\frac{\alpha_{1}}{1-\beta_{0}\left(\alpha_{1}\right)}<\frac{\alpha_{2}}{1-\beta_{0}\left(\alpha_{2}\right)} \\
& =\sup _{\alpha \in\left[\alpha_{1}, \alpha_{2}\right]}\left\{\frac{\alpha}{1-\beta_{0}(\alpha)}\right\}<p .
\end{aligned}
$$

Если положить $\gamma_{1}=\alpha_{1} /\left[1-\beta_{0}\left(\alpha_{1}\right)\right]$ и $\gamma_{2}=\alpha_{2} /\left[1-\beta_{0}\left(\alpha_{2}\right)\right]$, то для любого $\alpha \in\left[\alpha_{1}, \alpha_{2}\right], 1<\gamma_{1} \leqslant \alpha /\left[1-\beta_{0}(\alpha)\right] \leqslant \gamma_{2}<p$ и поэтому при $\beta=\beta_{0}(\alpha)$ из условия (5) следует (16). Сравнивая уравнения (9) и (18), получаем, что для любого $\alpha \in(0, p) z^{*}(\alpha)=z_{0}(\alpha)$, если $\beta=\beta_{0}(\alpha)$. Поэтому если $n, N \rightarrow \infty$ так, что выполнено условие $(5)$, то $S_{p}\left(z^{*}\right)=$ $S_{p}\left(z_{0}\right)=\beta_{0}^{-1}$ при условии $\beta=\beta_{0}$, и оценка (17) позволяет утверждать, что при $\beta=\beta_{0}$ и $m=\beta_{0} N \in N$ в случае $p \geqslant 2$ имеем:

$$
\begin{aligned}
\mathbf{P}\left\{\mu_{0}(p)=m\right\} & =\sqrt{\frac{\alpha-\left(z_{0}-\alpha\right)(p-\alpha)}{2 \pi N \Delta_{0}}}\left(1+O\left(\frac{1}{N}\right)\right) \\
& =\sqrt{\frac{1}{2 \pi N \sigma_{0}^{2}}}\left(1+O\left(\frac{1}{N}\right)\right)
\end{aligned}
$$

равномерно относительно $\alpha=\left[\alpha_{1}, \alpha_{2}\right]$, где $\Delta_{0}=\Delta_{z}$ при $\beta=\beta_{0}$ и

$$
\sigma_{0}^{2}=\frac{\Delta_{0}}{\alpha-\left(z_{0}-\alpha\right)(p-\alpha)}>0 \text {. }
$$

Далее будет показано, что оценка (22) - это частный случай локальной нормальной предельной теоремы для случайной величины $\mu_{0}(p)$. Из 
соотношения (23) и формул для величины $\Delta_{z}$, приведенных в формулировке теоремы 2 , при $\beta=\beta_{0}$ легко получить, что для любого $\alpha \in(0, p)$

$$
\sigma_{0}^{2}=\frac{\alpha \beta_{0}\left(1-\beta_{0}\right)}{\alpha-\left(z_{0}-\alpha\right)(p-\alpha)}\left(p+1+\left(1-\frac{p}{\alpha}\right) z_{0}-\frac{\alpha}{1-\beta_{0}}\right) .
$$

Если $p \geqslant 2$, то $\sigma_{0}^{2}>0$; если же $p=1$, то $\sigma_{0}^{2}=0$, поскольку в этом случае для любого $\alpha \in(0,1) z_{0}(\alpha)=\alpha /(1-\alpha)$ и $\beta_{0}=1-\alpha$. Заметим, что если $\alpha \in(0, p) u 1<\alpha /(1-\beta)<p$, mo при $\beta>\beta_{0}(\alpha) z^{*}>z_{0}(\alpha) u u^{*}>1, a$ при $\beta<\beta_{0}(\alpha) z^{*}<z_{0}(\alpha) u 0<u^{*}<1$.

Действительно, при $\beta=\beta_{0}(\alpha)$, как отмечено выше, имеем: $\forall \alpha \in$ $(0, p) z^{*}(\alpha)=z_{0}(\alpha)$, и поэтому $u^{*}=1$ в силу $(10)$. Если же $\beta>\beta_{0}(\alpha)$, то из (18) следует, что $\sum_{k=1}^{p}\left(k-\alpha /\left[1-\beta_{0}(\alpha)\right]\right)\left(z^{*}\right)^{k} / k !>0$; так как

$$
\sum_{k=1}^{p}\left(k-\frac{\alpha}{1-\beta_{0}(\alpha)}\right) \frac{z_{0}^{k}}{k !}=0
$$

и функция $h(z)=z S_{p}^{\prime}(z) /\left[S_{p}(z)-1\right]$ строго возрастает на интервале $(0,+\infty)$ от 1 до $p$, то в этом случае $z^{*}>z_{0}(\alpha)$, и поэтому

$$
\sum_{k=1}^{p} \frac{\left(z^{*}\right)^{k}}{k !}>\sum_{k=1}^{p} \frac{\left(z_{0}(\alpha)\right)^{k}}{k !}=\frac{1-\beta_{0}(\alpha)}{\beta_{0}(\alpha)}>\frac{1-\beta}{\beta},
$$

так что, согласно (19), $u^{*}>1$. Аналогично показывается, что в случае $\beta<\beta_{0}(\alpha) z^{*}<z_{0}(\alpha)$ и $0<u^{*}<1$.

Теорема 3. Пусть при условиях теоремы $2 \alpha_{2} /\left[1-\beta_{0}\left(\alpha_{2}\right)\right]<\gamma_{1}$. Тогда равномерно относительно $\alpha \in\left[\alpha_{1}, \alpha_{2}\right]$ и значений $\beta$, удовлетворяющих условию (16),

$$
\mathbf{P}\left\{\mu_{0}(p) \geqslant m\right\}=\frac{\mathbf{P}\left\{\mu_{0}(p)=m\right\}}{1-(1-\beta) /\left[\beta\left(S_{p}\left(z^{*}\right)-1\right)\right]}\left(1+O\left(\frac{1}{N}\right)\right) .
$$

Заметим, что при условиях (5) равномерно относительно $\alpha \in\left[\alpha_{1}, \alpha_{2}\right]$

$$
\mathbf{E} \mu_{0}(p)=N \frac{M_{n}(N-1, p)}{M_{n}(N, p)}=N \beta_{0}+O(1)
$$

(это равенство будет установлено ниже), и поэтому «порог» в выборе значений $\beta$ определяется отношением $\mathbf{E} \mu_{0}(p) / N=\beta_{0}+O(1 / N)$. Если $\alpha_{2} /\left[1-\beta_{0}\left(\alpha_{2}\right)\right]<\gamma_{1}$, то из (5) получаем, что при любом $\alpha \in\left[\alpha_{1}, \alpha_{2}\right]$ и при любых значениях $\beta$, удовлетворяющих условию (16), $\beta \geqslant \beta_{0}(\alpha)+C$, где $C>0$ - постоянная, и поэтому $\inf _{(\alpha, \beta)}\left\{u^{*}\right\}>1$. Отсюда нетрудно вывести, что

$$
\mathbf{P}\left\{\mu_{0}(p) \geqslant m\right\}=\mathbf{P}\left\{\mu_{0}(p)=m\right\} \frac{u^{*}}{u^{*}-1}\left(1+O\left(\frac{1}{N}\right)\right)
$$


равномерно относительно указанных значений $\alpha$ и $\beta$. Из этой формулы и (19) следует (25). Теорема 3 доказана.

Если, в частности, при условиях теоремы $3 \alpha$ и $\beta$ - постоянные, то

$$
0<\lim _{m, n, N \rightarrow \infty} \frac{\mathbf{P}\left\{\mu_{0}(p)=m\right\}}{\mathbf{P}\left\{\mu_{0}(p) \geqslant m\right\}}=1-\frac{1-\beta}{\beta\left(S_{p}\left(z^{*}\right)-1\right)}<1 .
$$

Теорема 4. Пусть при условиях теоремы $2 \gamma_{2}<\alpha_{1} /\left[1-\beta_{0}\left(\alpha_{1}\right)\right]$. Тогда равномерно относительно $\alpha \in\left[\alpha_{1}, \alpha_{2}\right]$ и значений $\beta$, удовлетворяющих условию (16),

$$
\mathbf{P}\left\{\mu_{0}(p) \leqslant m\right\}=\mathbf{P}\left\{\mu_{0}(p)=m\right\} \frac{1-\beta}{1-\beta S_{p}\left(z^{*}\right)}\left(1+O\left(\frac{1}{N}\right)\right) .
$$

Доказательство теоремы 4 проводится аналогично доказательству теоремы 3. Следует заметить, что если $\gamma_{2}<\alpha_{1} /\left[1-\beta_{0}\left(\alpha_{1}\right)\right]$, то при любом $\alpha \in\left[\alpha_{1}, \alpha_{2}\right]$ и при любых значениях $\beta$, удовлетворяющих условию (16), $\beta \leqslant \beta_{0}(\alpha)-C_{1}$, где $C_{1}>0$ - некоторая постоянная, и поэтому $\sup _{(\alpha, \beta)}\left\{u^{*}\right\}<1$ и существует постоянная $\eta \in(0,1)$ такая, что $1-u^{m+1}=1+O\left(\eta^{N \beta}\right)$ равномерно относительно переменных $z, u$, удовлетворяющих условиям $|z|=z^{*}>0$ и $0<|u|=u^{*}<1$. Из равенства

$$
\begin{aligned}
\mathbf{P}\left\{\mu_{0}(p) \leqslant m\right\}= & \frac{n !}{M_{n}(N, p)} \frac{1}{(2 \pi i)^{2}} \\
& \times \oint \oint \frac{(f(z, u))^{N}}{(1-u) z^{n+1} u^{m+1}}\left(1-u^{m+1}\right) d z d u
\end{aligned}
$$

так же, как и выше, получаем:

$$
\mathbf{P}\left\{\mu_{0}(p) \leqslant m\right\}=\frac{\mathbf{P}\left\{\mu_{0}(p)=m\right\}}{1-u^{*}}\left(1+O\left(\frac{1}{N}\right)\right)
$$

равномерно относительно указанных значений $\alpha$ и $\beta$. Из этой формулы и (19) получаем утверждение теоремы 4.

Если, в частности, при условиях теоремы $4 \alpha$ и $\beta$ - постоянные, то

$$
0<\lim _{m, n, N \rightarrow \infty} \frac{\mathbf{P}\left\{\mu_{0}(p)=m\right\}}{\mathbf{P}\left\{\mu_{0}(p) \leqslant m\right\}}=\frac{1-\beta S_{p}\left(z^{*}\right)}{1-\beta}<1 .
$$

Теорема 5. Пусть $n, N \rightarrow \infty$ так, ито выполнено условие (5). Тогда равномерно относительно $\alpha \in\left[\alpha_{1}, \alpha_{2}\right]$

$$
\mathbf{E} \mu_{0}(p)=N \beta_{0}+O(1), \quad \mathbf{D} \mu_{0}(p)=N \sigma_{0}^{2}+O(1)
$$

где величины $\beta_{0}$ и $\sigma_{0}^{2}$ определяются из соотношений (10) и (24).

Заметим, что при $p=1 \mathrm{D} \mu_{0}(1)=0$, если $n \leqslant N$, так как в этом случае $\mu_{0}(1)=N-n$ с вероятностью 1 , и $\mathbf{E} \mu_{0}(1)=N-n$. Поскольку 
при $p=1$ для любого $\alpha \in(0,1) \beta_{0}=1-\alpha$ и $\sigma_{0}^{2}=0$, то $\mathbf{E} \mu_{0}(1)=N \beta_{0}$ и $\mathbf{D} \mu_{0}(1)=N \sigma_{0}^{2}$. Пусть $p \geqslant 2$, тогда из (12) при $j=1$ и $j=2$ получаем:

$$
\begin{aligned}
\mathbf{E} \mu_{0}(p) & =N \frac{M_{n}(N-1, p)}{M_{n}(N, p)}, \\
\mathbf{E}\left(\mu_{0}(p)\left(\mu_{0}(p)-1\right)\right) & =N(N-1) \frac{M_{n}(N-2, p)}{M_{n}(N, p)} .
\end{aligned}
$$

Поскольку, согласно (4),

$$
M_{n}(N-1, p)=\frac{n !}{2 \pi i} \oint \frac{\left(S_{p}(z)\right)^{N-1}}{z^{n+1}} d z
$$

и $S_{p}\left(z_{0}\right)=\beta_{0}^{-1}$, то из $(8)$ следует, что при условиях теоремы 5 $M_{n}(N-1, p)=\beta_{0} M_{n}(N, p)(1+O(1 / N))$ равномерно относительно $\alpha \in\left[\alpha_{1}, \alpha_{2}\right]$, что с учетом (26) равносильно первому равенству в утверждении теоремы 5 . Далее, из (26) и (27) получаем

$$
\begin{aligned}
\mathbf{D} \mu_{0}(p)= & N(N-1) \frac{M_{n}(N-2, p)}{M_{n}(N, p)} \\
& +N \frac{M_{n}(N-1, p)}{M_{n}(N, p)}\left(1-N \frac{M_{n}(N-1, p)}{M_{n}(N, p)}\right) .
\end{aligned}
$$

Пусть при условиях доказываемой теоремы равномерно относительно $\alpha \in\left[\alpha_{1}, \alpha_{2}\right]$

$$
\begin{aligned}
M_{n}(N-j, p)= & \frac{n !}{\sqrt{2 \pi N\left(\alpha-\left(z_{0}-\alpha\right)(p-\alpha)\right)}} \\
& \times \frac{\beta_{0}^{-N+j}}{z_{0}^{n}}\left(1+\frac{\theta_{j}}{N}+O\left(\frac{1}{N^{2}}\right)\right) \quad(j=0,1,2) .
\end{aligned}
$$

Тогда из (28) можно вывести, что

$$
\mathbf{D} \mu_{0}(p)=N \beta_{0}\left(1-\beta_{0}+\beta_{0}\left(\theta_{0}+\theta_{2}-2 \theta_{1}\right)\right)+O(1)
$$

равномерно относительно $\alpha \in\left[\alpha_{1}, \alpha_{2}\right]$, и

$$
\sigma_{0}^{2}=\beta_{0}\left(1-\beta_{0}\right)+\beta_{0}^{2}\left(\theta_{0}+\theta_{2}-2 \theta_{1}\right) .
$$

Отсюда следует второе равенство в утверждении теоремы 5.

Теорема 6. Пусть $n, N \rightarrow \infty$ так, ито выполнено условие (5). Тогда при $p \geqslant 2$ равномерно относительно $\alpha \in\left[\alpha_{1}, \alpha_{2}\right]$ и челых положительных значений $m=\beta_{0} N+x \sqrt{N}$, для которых величина $x$ заключена в любом фиксированном конечном промежутке,

$$
\mathbf{P}\left\{\mu_{0}(p)=m\right\}=\frac{1}{\sqrt{2 \pi N \sigma_{0}^{2}}} \exp \left(-\frac{x^{2}}{2 \sigma_{0}^{2}}\right)\left(1+O\left(\frac{1}{\sqrt{N}}\right)\right),
$$

где величины $\beta_{0}$ и $\sigma_{0}^{2}$ определяются из соотношений (10) $u$ (24). 
Д ок а з а т е л с с т в о. При условии (5) формулу (30) можно получить из (17), полагая $\beta=\beta_{0}+x / \sqrt{N}$; можно показать, в частности, что тогда

$$
z^{*}=z_{0}\left(1+\frac{\alpha \beta_{0} x}{\left(\alpha-\left(z_{0}-\alpha\right)(p-\alpha)\right) \sigma_{0}^{2} \sqrt{N}}+O\left(\frac{1}{N}\right)\right)
$$

равномерно относительно $\alpha \in\left[\alpha_{1}, \alpha_{2}\right]$. Однако, чтобы избежать громоздких выкладок, предпочтительнее использовать метод, позволяющий свести изучение асимптотического поведения вероятности $\mathbf{P}\left\{\mu_{0}(p)=m\right\}$ к применению классической локальной нормальной предельной теоремы для последовательности независимых одинаково распределенных целочисленных случайных величин $[2$, с. 59]. Рассмотрим последовательность независимых одинаково распределенных случайных величин $\xi_{1}, \ldots, \xi_{N}, \ldots$, таких, что

$$
\mathbf{P}\left\{\xi_{1}=k\right\}=\frac{z_{0}^{k}}{k ! S_{p}\left(z_{0}\right)} \quad(k=0,1, \ldots, p) .
$$

Легко подсчитать, что $\mathbf{E} \xi_{1}=z_{0} S_{p-1}\left(z_{0}\right) / S_{p}\left(z_{0}\right)=\alpha$ в силу (9) и $\mathbf{D} \xi_{1}=\alpha(p+1-\alpha)-(p-\alpha) z_{0}>0$, поскольку $z_{0}<\alpha(1+1 /(p-\alpha))$, если $\alpha \in(0, p)$. Согласно $(8)$, локальной нормальной предельной теореме для последовательности независимых одинаково распределенных случайных величин $\xi_{1}, \ldots, \xi_{N}, \ldots$, если $n, N \rightarrow \infty$ так, что выполнено условие (5), то равномерно относительно $\alpha \in\left[\alpha_{1}, \alpha_{2}\right]$

$$
\begin{aligned}
& \mathbf{P}\left\{\xi_{1}+\cdots+\xi_{N}=n\right\}=\frac{M_{n}(N, p)}{n !} z_{0}^{n} \beta_{0}^{N} \\
& \quad=\frac{1}{\sqrt{2 \pi N\left(\alpha(p+1-\alpha)-(p-\alpha) z_{0}\right)}}\left(1+O\left(\frac{1}{N}\right)\right)
\end{aligned}
$$

(см. [12]). Далее, рассмотрим последовательность независимых одинаково распределенных случайных величин $\xi_{1}^{(0)}, \ldots, \xi_{N-m}^{(0)}, \ldots$ таких, что

$$
\mathbf{P}\left\{\xi_{1}^{(0)}=k\right\}=\mathbf{P}\left\{\xi_{1}=k \mid \xi_{1} \neq 0\right\}=\frac{z_{0}^{k}}{k !\left(S_{p}\left(z_{0}-1\right)\right)} \quad(k=1,2, \ldots, p) .
$$

Нетрудно показать, что $\mathbf{E} \xi_{1}^{(0)}=\alpha /\left(1-\beta_{0}\right)$ и

$$
\mathbf{D} \xi_{1}^{(0)}=\frac{(p+1) \alpha-\alpha^{2} /\left(1-\beta_{0}\right)-(p-\alpha) z_{0}}{1-\beta_{0}}>0
$$

при $p \geqslant 2$.

При условиях теоремы 6

$$
\frac{n}{N-m}=\frac{\alpha}{1-\beta_{0}-x / \sqrt{N}}=\mathbf{E} \xi_{1}^{(0)}\left(1+O\left(\frac{1}{\sqrt{N}}\right)\right)
$$


равномерно относительно $\alpha \in\left[\alpha_{1}, \alpha_{2}\right]$, поскольку величина $\mathbf{E} \xi_{1}^{(0)}$ ограничена. Поэтому, согласно локальной нормальной предельной теореме для последовательности независимых случайных величин $\xi_{1}^{(0)}, \ldots, \xi_{N-m}^{(0)}, \ldots$, имеем равномерно по $\alpha \in\left[\alpha_{1}, \alpha_{2}\right]$ :

$$
\begin{array}{r}
\mathbf{P}\left\{\xi_{1}^{(0)}+\cdots+\xi_{N-m}^{(0)}=n\right\}=\frac{1}{\sqrt{2 \pi N\left(1-\beta_{0}\right) \mathbf{D} \xi_{1}^{(0)}}} \\
\quad \times \exp \left(-\frac{\alpha^{2} x^{2}}{2\left(1-\beta_{0}\right)^{3} \mathbf{D} \xi_{1}^{(0)}}\right)\left(1+O\left(\frac{1}{\sqrt{N}}\right)\right) .
\end{array}
$$

При условиях теоремы 6, согласно локальной теореме МуавраЛaпraca,

$$
\begin{aligned}
C_{N}^{m} \beta_{0}^{m}\left(1-\beta_{0}\right)^{N-m}= & \frac{1}{\sqrt{2 \pi N \beta_{0}\left(1-\beta_{0}\right)}} \\
& \times \exp \left(-\frac{x^{2}}{2 \beta_{0}\left(1-\beta_{0}\right)}\right)\left(1+O\left(\frac{1}{\sqrt{N}}\right)\right)
\end{aligned}
$$

равномерно по $\alpha \in\left[\alpha_{1}, \alpha_{2}\right]$. Поскольку $\mathbf{P}\left\{\xi_{1}=0\right\}=1 / S_{p}\left(z_{0}\right)=\beta_{0}$ в силу (10), то аналогично [2, с. 58] можно утверждать, что

$$
\mathbf{P}\left\{\mu_{0}(p)=m\right\}=C_{N}^{m} \beta_{0}^{m}\left(1-\beta_{0}\right)^{N-m} \frac{\mathbf{P}\left\{\xi_{1}^{(0)}+\cdots+\xi_{N-m}^{(0)}=n\right\}}{\mathbf{P}\left\{\xi_{1}+\cdots+\xi_{N}=n\right\}}
$$

Из формул (24), (31)-(35) следует (30). Теорема 6 доказана.

Следствие 1. При условиях теоремы 6 случайная величина $\left[\mu_{0}(p)-\beta_{0} N\right] / \sqrt{N \sigma_{0}^{2}}$ распределена в пределе по стандартному нормальному закону.

\section{3. Случай одинаковых частиц.}

Теорема 7. Пусть $n, N \rightarrow \infty$ так, что өыполнено условие (5). Тогда равномерно относительно $\alpha \in\left[\alpha_{1}, \alpha_{2}\right]$

$$
f_{n}(N, p)=\frac{\gamma_{0}^{-N} x_{0}^{-n}}{\sqrt{2 \pi N \gamma_{0} \sum_{k=1}^{p} k(k-\alpha) x_{0}^{k}}}\left(1+O\left(\frac{1}{N}\right)\right)
$$

где $x=x_{0}-$ единственный вещественный положительный корень уравнения

$$
\sum_{k=0}^{p}(k-\alpha) x^{k}=0
$$

$u$

$$
\gamma_{0}=\left(\sum_{k=0}^{p} x_{0}^{k}\right)^{-1}
$$


Обоснование формулы (36) с учетом соотношений (37) и (38) проводится аналогично доказательству теоремы 1 , исходя из (7); это обоснование имеется в [10].

Факториальные моменты случайной величины $\xi_{0}(p)$ определяются равенствами $[3$, с. 126$]$

$$
\mathbf{E}\left(\xi_{0}(p)\right)_{j}=N(N-1) \cdots(N-j+1) \frac{f_{n}(N-j, p)}{f_{n}(N, p)}
$$

$\left(j=0,1, \ldots, N ; f_{n}(0, p)=0\right)$. Из (7) и (39) следует, что производящая функция распределения вероятностей для $\xi_{0}(p)$ равна

$$
P_{\xi_{0}(p)}(u)=\left(f_{n}(N, p)\right)^{-1} \frac{1}{2 \pi i} \oint \frac{\left(u+\sum_{k=1}^{p} z^{k}\right)^{N}}{z^{n+1}} d z .
$$

Из (40) получаем

$$
\mathbf{P}\left\{\xi_{0}(p)=m\right\}=\left(f_{n}(N, p)\right)^{-1} \frac{1}{(2 \pi i)^{2}} \oint \oint \frac{(g(z, u))^{N}}{z^{n+1} u^{m+1}} d z d u
$$

$m=0,1, \ldots, N$, где

$$
g(z, u)=u+\sum_{k=1}^{p} z^{k}
$$

Положим

$$
T_{r}(x)=\sum_{k=0}^{r} x^{k} \quad(x>0 ; r=0,1,2, \ldots)
$$

Теорема 8. Пусть $n, N \rightarrow \infty$ так, что выполнено условие (5); $p \geqslant 2$ и $m=\beta N \rightarrow \infty$ так, что справедливо (16). Тогда равномерно относительно $\alpha \in\left[\alpha_{1}, \alpha_{2}\right]$ и значений $\beta$, удовлетворяюших условию (16),

$$
\begin{aligned}
\mathbf{P}\left\{\xi_{0}(p)=m\right\}= & \sqrt{\frac{\gamma_{0} \sigma}{2 \pi N \Delta_{x}}}\left(\frac{x_{0}}{x^{*}}\right)^{n} \\
& \times \gamma_{0}^{N} \beta^{-m}\left(\frac{T_{p}\left(x^{*}\right)-1}{1-\beta}\right)^{N-m}\left(1+O\left(\frac{1}{N}\right)\right)
\end{aligned}
$$

где $x=x_{0}-$ единственный вещественный положительный корень уравнения (37), $x=x^{*}$ - единственный вещественный положительный хорень уравнения $\sum_{k=1}^{p}(k-\alpha /(1-\beta)) x^{k}=0$,

$$
\begin{gathered}
\sigma=\sum_{k=1}^{p} k(k-\alpha) x_{0}^{k}>0 \\
\Delta_{x}=\beta(1-\beta)^{2}\left(\frac{\sum_{k=1}^{p} k^{2}\left(x^{*}\right)^{k}}{T_{p}\left(x^{*}\right)-1}-\frac{\alpha^{2}}{(1-\beta)^{2}}\right)>0
\end{gathered}
$$

значения $\gamma_{0}, T_{p}\left(x^{*}\right)$ определяются соеласно $(38),(43)$. 
Д ок аз а т е ль с т в о фо р м у лы (44) проводится аналогично обоснованию формулы (17) с учетом соотношений (41) и (42).

Теорема 9. Пусть при условиях теоремы $8 \alpha_{2} /\left[1-\gamma_{0}\left(\alpha_{2}\right)\right]<\gamma_{1}$. Тогда равномерно относительно $\alpha \in\left[\alpha_{1}, \alpha_{2}\right]$ и значений $\beta$, удовлетворяюиих условию (16),

$$
\mathbf{P}\left\{\xi_{0}(p) \geqslant m\right\}=\frac{\mathbf{P}\left\{\xi_{0}(p)=m\right\}}{1-(1-\beta) /\left[\beta\left(T_{p}\left(x^{*}\right)-1\right)\right]}\left(1+O\left(\frac{1}{N}\right)\right) .
$$

Если, в частности, при условиях теоремы $9 \alpha$ и $\beta$ - постоянные, то

$$
0<\lim _{m, n, N \rightarrow \infty} \frac{\mathbf{P}\left\{\xi_{0}(p)=m\right\}}{\mathbf{P}\left\{\xi_{0}(p) \geqslant m\right\}}=1-\frac{1-\beta}{\beta\left(T_{p}\left(x^{*}\right)-1\right)}<1 .
$$

Теорема 10. ПІусть при условиях теоремы $8 \gamma_{2}<\alpha_{1} /\left[1-\gamma_{0}\left(\alpha_{1}\right)\right]$. Тогда равномерно относительно $\alpha \in\left[\alpha_{1}, \alpha_{2}\right]$ и значений $\beta$, удовлетворяющих условию (16),

$$
\mathbf{P}\left\{\xi_{0}(p) \leqslant m\right\}=\mathbf{P}\left\{\xi_{0}(p)=m\right\} \frac{1-\beta}{1-\beta T_{p}\left(x^{*}\right)}\left(1+O\left(\frac{1}{N}\right)\right) .
$$

Если, в частности, при условиях теоремы $10 \alpha$ и $\beta$ - постоянные, то

$$
0<\lim _{m, n, N \rightarrow \infty} \frac{\mathbf{P}\left\{\xi_{0}(p)=m\right\}}{\mathbf{P}\left\{\xi_{0}(p) \leqslant m\right\}}=\frac{1-\beta T_{p}\left(x^{*}\right)}{1-\beta}<1 .
$$

Теоремы 9 и 10 доказываются аналогично теоремам 3 и 4.

Теорема 11. Пусть $n, N \rightarrow \infty$ так, ито выполнено условие (5). Тогда равномерно относительно $\alpha \in\left[\alpha_{1}, \alpha_{2}\right]$

$$
\mathbf{E} \xi_{0}(p)=N \gamma_{0}+O(1), \quad \mathbf{D} \xi_{0}(p)=N \sigma_{1}^{2}+O(1),
$$

2de

$$
\sigma_{1}^{2}=\gamma_{0}\left(1-\gamma_{0}-\frac{\alpha^{2}}{\sigma}\right)
$$

и величины $\gamma_{0}$ и $\sigma$ определяются из соотношений (38) $u$ (45).

Теорема 11 доказывается, исходя из (36) и (39) с использованием того же метода, который применялся при доказательстве теоремы 5. Заметим, что при $p=1 \sigma_{1}^{2}=0$, а при $p \geqslant 2 \sigma_{1}^{2}>0$, согласно неравенству Коши-Буняковского.

Теорема 12. Пусть $n, N \rightarrow \infty$ так, что выполнено условие (5). Тогда при $p \geqslant 2$ равномерно относительно $\alpha \in\left[\alpha_{1}, \alpha_{2}\right]$ и челых положительных значений $m=\gamma_{0} N+x \sqrt{N}$, для которых величина $x$ заключена в любом фиксированном конечном промежутке,

$$
\mathbf{P}\left\{\xi_{0}(p)=m\right\}=\frac{1}{\sqrt{2 \pi N \sigma_{1}^{2}}} \exp \left(-\frac{x^{2}}{2 \sigma_{1}^{2}}\right)\left(1+O\left(\frac{1}{\sqrt{N}}\right)\right),
$$

где величины $\gamma_{0}$ и $\sigma_{1}^{2}$ определяются из соотношений (38) $и$ (46). 
При доказательстве теоремы 12 применяется тот же метод, с использованием которого устанавливалось равенство (30). При этом предполагается, что соответствующие случайные величины $\xi_{1}, \ldots, \xi_{N}, \ldots$ имеют усеченное геометрическое распределение с параметром $x_{0}$, т.е.

$$
\mathbf{P}\left\{\xi_{i}=k\right\}=\frac{x_{0}^{k}}{T_{p}\left(x_{0}\right)} \quad(k=0,1, \ldots, p, i=1,2, \ldots, N, \ldots) .
$$

Заметим, что теоремы 11 и 12 сформулированы в [11].

Следствие 2. При условиях теоремы 12 случайная величина $\left[\xi_{0}(p)-\gamma_{0} N\right] / \sqrt{N \sigma_{1}^{2}}$ распределена в пределе по стандартному нормальному закону.

\section{СПИСОК ЛИТЕРАТУРЫ}

1. Феллер В. Введение в теорию вероятностей и ее приложения. Т. 1. М.: Мир, $1967,752 \mathrm{c}$.

2. Колиия B. Ф., Севастьямов Б. А., Чистяков В. П. Случайные размешения. М.: Наука, 1976, 223 c.

3. Риордак Дж. Введение в комбинаторный анализ. М.: ИЛ, 1963, 287 с.

4. Тимашев $A$. $H$. Многомерные локальные теоремы о больших уклонениях в равновероятной схеме размещения. - Дискретн. матем., 1990, т. 2, в. 2, с. 145-149.

5. Тимаиев $A . H$. Многомерная интегральная теорема о больших уклонениях в равновероятной схеме размещения. - Дискретн. матем., 1992, т. 4, в. 4, с. 74-81.

6. Труков $A . H$. Предельные теоремы в задаче о размещени одинаковых частиц по различным ячейкам. - Труды Матем. ин-та им. В.А. Стеклова, 1986, т. 177, c. $147-166$.

7. Тимащев A. H. Теоремы о больших уклонениях в схеме размещения одинаковых частиц по различным ячейкам. - Дискретн. матем., 1993, т. 5, в. 4, с. 29-42.

8. Федорюк М.В. Метод перевала. М.: Наука, 1977.

9. Олвер $Ф$. Введение в асимптотические методы и специальные функции. М.: Наука, 1978.

10. Тимашев $A . H$. Пууассоновское предельное распределение числа пустых ячеек в обобщенной равновероятной схеме размещения частиц комплектами. - Дискретн. матем., 1995, т. 7 , в. 2 , с. $140-145$.

11. Тимашев $A$. Н. Локальная нормальная теорема в схеме размешений одинаковых частиц по различным ячейкам при ограниченных объемах ячеек. - Обозрение прикл. промышл. матем., 1997, т. 4, в. 3, с. 411-412.

12. Holst $L$. On numbers related to partition of unlike objects and occupancy problems. - European J. Combin., 1981, v. 2, p. 231-237.

Поступила в редакцию 29.XII.1997

Исправленный вариант

2.XII.1998 